\title{
For Premature Thelarche and Premature Adrenarche, the Case for Waiting before Testing
}

\author{
Paul B. Kaplowitz \\ Division of Endocrinology, Children's National Hospital, Washington, DC, USA
}

\section{Keywords}

Premature thelarche - Premature adrenarche $\cdot$ Central precocious puberty · Nonclassical congenital adrenal hyperplasia - Low-value care

\section{Introduction}

Premature thelarche (PT) and premature adrenarche (PA) are the 2 most common variations of early pubertal development seen by pediatric endocrinologists. Typical cases are usually easy to recognize based on their clinical presentation and do not require specific therapy. While more experienced endocrine providers often do not order any tests and opt for follow-up either in person or by the referring physician, it is common for some initial testing to be performed to make sure that something more serious is not missed. The concern is usually either central precocious puberty (CPP) in cases of early breast development, or some form of congenital adrenal hyperplasia $(\mathrm{CAH})$ in cases of early pubic hair development. I will make the case, supported by recent studies and my personal experience, that in typical cases without red flags, testing adds unnecessary expense and sometimes results in an erroneous diagnosis leading to inappropriate initiation of treatment, or a correct diagnosis for which treatment may not be needed.

$\begin{aligned} & \text { karger@karger.com } \\ & \text { www.karger.com/hrp }\end{aligned}$
Karger $\%$

\section{Premature Thelarche}

PT refers to the appearance of very early breast development in girls, usually in the first 2 years of life and in most cases before age 3 . While the etiology is poorly understood, the course in young girls is fairly typical. Breasts are typically Tanner stage 2 but occasionally stage 3 ; they rarely progress significantly over time and in many cases (60\% in one study) [1] regress spontaneously. In a series of 156 girls seen at the author's hospital over a 4-year period, the mean age of parent-reported appearance of breast tissue was 7.4 months and the mean age at the first visit was 19 months; thus, on average, approximately 1 year had elapsed between onset and endocrine evaluation [2]. Eleven percent had a height $>90$ th \%ile, confirming that rapid growth is not a common feature of PT. Most of those tested had low luteinizing hormone $(\mathrm{LH})<0.3$ $\mathrm{mIU} / \mathrm{L}$ and estradiol below the assay detection limit of 20 $\mathrm{pg} / \mathrm{mL}$ (73 pmol/L), but of the ones with mildly elevated levels, none had both an elevated LH and estradiol.

There is some dispute as to how often PT progresses to CPP. In a study of 139 girls, this appeared to be common, with CPP occurring in 13\% [3] irrespective of age at PT presentation or clinical course, while in another study, $7 / 38$ (18\%) followed at least until age 8 had CPP (1), but in both studies, several years usually elapsed before a CPP diagnosis was made. However, in a larger Italian study of 
450 girls under age 3 initially diagnosed with PT, only 9 (2\%) were confirmed to have CPP during follow-up (4). In the author's personal experience over a 35-year period encompassing over 500 girls, no girls under age 3 who were diagnosed with PT were later diagnosed with CPP.

Part of the problem with defining CPP is that in many cases, it is diagnosed hormonally based on the peak LH of $>5 \mathrm{mIU} / \mathrm{mL}$, considered to be the gold standard. However, 2 recent studies make it clear that $\mathrm{LH}$ response to gonadotropin-releasing hormone $(\mathrm{GnRH})$ is not reliable in diagnosing CPP in girls under age $3[4,5]$. In one study [5], which included only girls found not to have progressed during follow-up of at least 1 year, the baseline median LH was $0.29(0.10-0.74) \mathrm{IU} / \mathrm{L}$, baseline median follicle-stimulating hormone (FSH) was 4.96 (3.18-7.05) IU/L, the peak median $\mathrm{LH}$ after GnRH stimulation was $5.75(3.31-8.58) \mathrm{IU} / \mathrm{L}$, and the peak mean FSH was 40.4 IU/L. Among the 35 patients, $33.3 \%(n=10)$ had baseline $\mathrm{LH}>0.3 \mathrm{IU} / \mathrm{L}, 67 \%(n=20)$ had peak $\mathrm{LH}>5 \mathrm{IU} / \mathrm{L}$, and $16.6 \%(n=5)>10 \mathrm{IU} / \mathrm{L}$. The peak LH/FSH ratio was $0.17 \pm 0.09$ and $\leq 0.43$ in all. Thus, the LH/FSH ratio was much more accurate in confirming a diagnosis of PT than the peak LH [5].

I will provide one example which demonstrates how overreliance on testing can lead to unnecessary treatment. A 2 4/14 year old girl with breast development since age 6 months was seen by an endocrine provider. Screening labs performed by the referring provider showed $\mathrm{LH}$ $0.18 \mathrm{IU} / \mathrm{L}, \mathrm{FSH} 9.2 \mathrm{IU} / \mathrm{L}$, estradiol $<3 \mathrm{pg} / \mathrm{mL}$, and bone age 3 years. Length was at the 50th \%ile. While initially the endocrine provider felt this was PT, during a $\mathrm{GnRH}$ stimulation test, the peak LH was 6.8 and peak FSH 36, which was felt to be diagnostic of CPP. At next visit 3 months later, there was slight growth acceleration. A brain magnetic resonance imaging (MRI) was ordered which was normal. The child was started on a monthly $\mathrm{GnRH}$ agonist and then scheduled for a histrelin implant which was placed at age 2 years 8 months. At her last follow-up visit at age 8 , she had received several more implants, had no progression of puberty, and in fact the breast tissue had regressed (as occurs spontaneously in $60 \%$ of PT patients according to one study), but the parents became concerned about the increasingly high charges for the implants. Since little monitoring was performed before treatment was initiated, one cannot be certain that this was PT and not CPP. However, the age of onset, the lack of significant progression before referral, the FSH-predominant response, and the negative MRI all strongly suggest PT. If testing had been deferred, the likelihood is that the child would not have been treated with expensive implants for over 5 years. CPP is much more likely in a 5-8 year old girl presenting with breast development, but even then, unless breast development has already reached Tanner stage 3 , a period of monitoring is recommended prior to making a decision to treat [6].

\section{Premature Adrenarche}

PA is generally diagnosed in girls with pubic hair but no breast development before age 8 and boys with pubic hair but no genital enlargement before age 9, with few cases diagnosed before age 3 . It is due to an earlier than normal timing of adrenarche, during which adrenal androgen production, particularly dehydroepiandrosterone (DHEA) and DHEA-sulfate (DHEA-S) increase. The main commonly abnormal lab test is DHEA-S, which is elevated for age but appropriate for the stage of pubic hair development. These children tend to be taller than average, and a few have accelerated growth, but other than the genital hair and often an adult-type axillary odor, they otherwise have a normal exam. It was stated in a recent review that "PA is diagnosis of exclusion, as true centrally mediated precocious puberty, congenital adrenal hyperplasia $(\mathrm{CAH})$, exogenous androgen exposure, and androgen-secreting tumors must be ruled out" [7]. However, the rare tumor can usually be excluded on clinical grounds, since they generally present earlier than typical PA (i.e., before age 3 ) with rapid growth, abundant pubic hair, and signs of virilization including genital enlargement [8]. CPP, unlike PA, is accompanied by breasts in girls and genital enlargement in boys. Thus, nonclassical or late-onset $\mathrm{CAH}$ (NC-CAH) is the most common alternative diagnosis, with studies finding that approximately $5 \%$ of PA patients have hormonal evidence of NC-CAH based on ACTH testing [9, 10]. One such study [9] found that $4 \%$ of 238 subjects with early pubic hair had NC$\mathrm{CAH}$ based on ACTH-stimulated 17-hydroxyprogesterone (17-OHP), but that all had random 17-OHP of at least $6 \mathrm{nmol} / \mathrm{L}(200 \mathrm{ng} / \mathrm{dL})$, which makes ACTH stimulation testing unnecessary in most cases.

Many providers order testing for all children with a clinical diagnosis of PA which typically includes DHEA$\mathrm{S}$, testosterone, and 17-OHP, as well as a bone age. Bone age advancement is considered by many to be a good way to identify children with pathological causes of PA. However, a retrospective study at my institution of 266 clinically diagnosed PA patients found that of the 121 who had a bone age exam, it was advanced by 2 or more years in $30 \%$, and this did not predict a finding of NC-CAH or 
other serious diagnoses [11]. Those with very advanced bone age were on average significantly taller with higher BMI than those with bone age advanced by less than 1 year, and DHEA-S levels were slightly higher in the very advanced BA group (mean DHEA-S 2,347 vs. 1,716 $\mathrm{nmol} / \mathrm{L}$ [86.3 vs. $63.1 \mu \mathrm{g} / \mathrm{dL}$ ], $p=0.03$ ), but 17-OHP levels and testosterone levels were similar between the 2 groups. One patient, a 6-year-old male with extreme tall stature (height SD + 3.1 SD), very rapid growth, a BA of 12 years, and an unstimulated $17-\mathrm{OHP}$ of $131 \mathrm{nmol} / \mathrm{L}(4,320 \mathrm{ng} /$ $\mathrm{dL}$ ), was identified with 21-hydroxylase deficiency CAH. Based on his growth pattern, this child had good reason to be tested. Thus, it was suggested that for most patients with PA and no red flags based on growth acceleration or genital findings other than pubic hair, a bone age is not necessary. In fact, in my experience, the finding of an advanced bone age by 2 years or more sometimes results in much concern and more extensive testing, including studies more appropriate for evaluation of CPP (including in a few cases, a brain MRI), with high cost and no clinical benefit.

Another factor which should be considered in the decision to test is the ethnic/racial background of the child. While NC-CAH is common in certain ethnic groups (e.g., Ashkenazi Jews), it appears to be less common to rare in African-American (AA) children. This was recently confirmed in a study from Alabama of 273 patients with PA, of whom $77 \%$ were AA but the 3 who were diagnosed with $\mathrm{CAH}$ ( 1 classic and $2 \mathrm{NC}-\mathrm{CAH}$ ) were all white [12]. The authors suggest adjusting screening protocols to reflect this finding, which would greatly reduce testing in children with PA since the majority affected at most centers in the USA are AA. This undoubtedly is related to the finding that 3 times as many AA versus white 7 - and 8 -year-old girls have pubic hair [13], yet the accepted definition of PA is onset before age 8 regardless of racial/ ethnic background.

An important question is whether screening for NC$\mathrm{CAH}$ should be performed in all children with PA due to concerns about missing a potentially treatable condition. While some children with NC-CAH clearly have clinical and growth evidence of hyperandrogenism and may benefit from treatment with glucocorticoids, many do not. Their height may be similar to children with PA and normal ACTH testing, though they tend to present at a younger age, be thinner and have a more advanced bone age [10]. While not testing all children with PA would likely miss a few children with $\mathrm{NC}-\mathrm{CAH}$, there is no research showing that children who are clinically indistinguishable from those with normal 17-OHP levels benefit from treatment in terms of prevention of more severe manifestations of androgen excess and short adult stature. One recent European multicenter study looked retrospectively at a group of $192 \mathrm{NC}-\mathrm{CAH}$ patients mostly treated with glucocorticoids who achieved adult heights only modestly below the normal range. However, the smaller untreated group ( $11 \%$ of the total) achieved normal and slightly greater adult heights than the treated group ( $p=0.053)$ [14]. While it is likely that the untreated group had a milder presentation as they were significantly older at diagnosis, it is clear that withholding treatment of NC-CAH does not inevitably lead to rapidly advancing bone age and short stature. However, no long-term randomized studies of treatment versus observation of children with NC-CAH have been reported, so conclusive evidence on the benefits of treatment is lacking.

One should also consider the possible harms of treating NC-CAH. I was consulted on an 8.5-year-old girl from an Ashkenazi Jewish family who had axillary odor starting at 7.5 years and pubic hair starting at 8 years. Height was 35th \%ile, and bone age was advanced 2 years. Her DHEA-S was 39, testosterone 13, and random 17OHP 3,500 ng/dL with an increase to 5,590 ng/dL after ACTH stimulation testing. Genotyping revealed that the girl, her younger 7-year-old sister who also had pubic hair, and the mother all tested homozygous for a common mutation associated with NC-CAH (V281L), and the father was a carrier. The mother was $5^{\prime} 2^{\prime \prime}$, slightly below her target height of $5^{\prime} 4^{\prime \prime}$, and was obviously fertile. Their endocrine provider recommended treatment with hydrocortisone at a dose of $13 \mathrm{mg} / \mathrm{m}^{2} /$ day, and at subsequent visits, their growth slowed and weight gain increased. I suggested stopping hydrocortisone, following which linear growth improved and weight gain slowed. Thus, one cannot assume that treatment of NC-CAH has no risks, which may include not just slower growth, but suppression of the hypothalamic-pituitary-adrenal axis. While the cost of the hydrocortisone is minimal, the costs of frequent follow-up visits and hormonal testing can be substantial.

\section{Conclusion}

For both PT and PA, the majority of cases are easily recognized on clinical grounds based on the age of onset ( $<3$ years for most cases of PT and 3-8 years for most cases of PA), their nonprogressive or slowly progressive course, and their lack of significant growth acceleration. 
It is important to be aware of the especially low risk of NC-CAH in AA children. A careful explanation of the likely benign nature should be given to the parents, and testing can be deferred pending monitoring by either the endocrine provider or the primary care provider. Some may argue that failing to test on the first visit is problematic if the patient progresses and fails to return for followup. If the endocrine provider chooses not to schedule a follow-up visit, it is critical to detail in the letter to the referring provider (ideally with a copy to the parents) the reasons (rapid growth and/or progression) that should prompt a return visit. It is acknowledged that a small proportion of children with early breast and/or pubic hair development have serious conditions requiring testing and treatment, and those usually have red flags based on their growth and physical exam. However, monitoring should identify the rare patient without red flags, usually within a year. Delaying testing while reassuring parents will decrease parental anxiety, reduce unnecessary testing, and prevent inappropriate or unnecessary treatments.

\section{Statement of Ethics}

No study approval and statement of ethics are needed, as this article presents no new research findings but is mainly a review of previously published work.

\section{Conflict of Interest Statement}

The author has no conflicts of interest to declare.

\section{Funding Sources}

The author did not receive any funding.

\section{References}

1 Volto C, Bernasconi S, Cisternino M, Buzi F, Street ME, Da Milana AM. Isolated premature thelarche and thelarche variant: clinical and auxological follow-up of 119 girls. J Endocrinol Invest. 1998 Mar;21(3):180-3.

2 Kaplowitz PB, Mehra R. Clinical characteristics of children referred for signs of early puberty before age 3. J Pediatr Endocrinol Metab. 2015 Sep 1;28(9-10):1139-44.

3 De Vries L, Guz-Mark A, Lazar L, Reches A, Phillip M. Premature thelarche: age at presentation affects clinical course but not clinical characteristics or risk to progress to precocious puberty. J Pediatr. 2010;156(3):466-71.

4 Bizzarri C, Spadoni GL, Bottaro G, Montanari G, Giannone G, Cappa M, et al. The response to gonadotropin releasing hormone $(\mathrm{GnRH})$ stimulation test does not predict the progression to true precocious puberty in girls with onset of premature thelarche in the first three years of life. J Clin Endocrinol Metab. 2014; 99(2):433-9.
5 Seymen Karabulut G, Atar M, Jones FMC, Hatun S. Girls with premature thelarche younger than 3 years of age might have stimulated $\mathrm{LH}$ greater than $10 \mathrm{IU} / \mathrm{L}$. J Clin Res Pediatr Endocrinol. 2020 Nov 25;12(4):377-82.

6 Carel JC, Eugster EA, Rogol A, Ghizzoni L, Palmert MR, Antoniazzi F, et al. Consensus statement on the use of gonadotropin-releasing hormone analogs in children. Pediatrics. 2009 Apr;123(4):e752-62.

7 Novello L, Speiser PW. Premature adrenarche. Pediatr Ann. 2018 Jan 1;47(1):e7-11.

8 Paris F, Kalfa N, Philibert P, Jeandel C, Gaspari L, Sultan C. Very premature pubarche in girls is not a pubertal variant. Hormones. 2012 Jul-Sep;11(3):356-60.

9 Armengaud JB, Charkaluk ML, Trivin C, Tardy V, Bréart G, Brauner R, et al. Precocious pubarche: distinguishing late-onset congenital adrenal hyperplasia from premature adrenarche. J Clin Endocrinol Metab. 2009;94(8): 2835-40.

10 Bizzarri C, Crea F, Marini R, Benevento D, Porzio $\mathrm{O}$, Ravà $\mathrm{L}$, et al. Clinical features suggestive of non-classical 21-hydroxylase deficiency in children presenting with precocious pubarche. J Pediatr Endocrinol Metab. 2012; 25(11-12):1059-64.
11 Desalvo DJ, Mehra R, Vaidyanathan P, Kaplowitz PB. In children with premature adrenarche, bone age advancement by 2 or more years is common and generally benign. J Pediatr Endocrinol Metab. 2013;26(3-4):21521.

12 Foster C, Diaz-Thomas A, Lahoti A. Low prevalence of organic pathology in a predominantly black population with premature adrenarche: need to stratify definitions and screening protocols. Int J Pediatr Endocrinol. 2020;2020:5

13 Biro FM, Galvez MP, Greenspan LC, Succop PA, Vangeepuram N, Pinney SM, et al. Pubertal assessment method and baseline characteristics in a mixed longitudinal study of girls. Pediatrics. 2010;126(3):e583.

14 Wasniewska MG, Morabito LA, Baronio F, Einaudi S, Salerno M, Bizzarri C, et al. Growth trajectory and adult height in children with nonclassical congenital adrenal hyperplasia. Horm Res Paediatr. 2020;93(3):173-81. 